\title{
THE SPATIAL PATTERN CHARACTERISTICS OF SOIL NUTRIENTS AT THE FIELD SCALE
}

\author{
Yujian Yang *, Jianhua Zhu, Xueqin Tong, Dianchang Wang \\ S\&T Information Engineering Research Center of Shandong Academy of Agricultural \\ Science, Jinan, Shandong Province, P. R. China 250100 \\ * Corresponding author, Address: S\&T Information Engineering Research Center, Number 28 \\ Sangyuan Road, Licheng District of Jinan, 250100, Shandong Province, P. R. China, Tel: \\ +86-531-83179076, Fax:+86-531-83179821,Email:xxzx_yyj@126.com
}

Abstract: This paper took the wheat field of typical alluvial soil area as the research site, aimed to explore the spatial pattern of the soil nutrients, such as soil available potassium, soil available phosphorus, soil organic matter (SOM) and soil alkali hydrolysable nitrogen. With the help of DGPS, a total of 104 topsoil $(0-20 \mathrm{~cm})$ samples were collected. Based on classical statistics, research results indicated the average content of soil available potassium was $287.476 \mathrm{~g} / \mathrm{kg}$, the high value was $483.433 \mathrm{~g} / \mathrm{kg}$, the low value was $119.435 \mathrm{~g} / \mathrm{kg}$. The average content of soil available phosphorus was $54.228 \mathrm{~g} / \mathrm{kg}$, the high value was $293.13 \mathrm{~g} / \mathrm{kg}$, the low value was $18.77 \mathrm{~g} / \mathrm{kg}$. The average content of SOM was $36.901 \mathrm{~g} / \mathrm{kg}$, the high value was $47.420 \mathrm{~g} / \mathrm{kg}$, the low value was $21.110 \mathrm{~g} / \mathrm{kg}$. The average content of soil alkali hydrolysable nitrogen was $66.444 \mathrm{~g} / \mathrm{kg}$, the high value was $166.474 \mathrm{~g} / \mathrm{kg}$, the low value was $29.846 \mathrm{~g} / \mathrm{kg}$. Geostatistical analysis, combined with geographical information system(GIS), the range, nugget and sill of soil nutrients characteristics were calculated, semivariogram model was established, ordinary kriging was applied, and the spatial distributing map of soil nutrients was drawn. It is shown that the semivariogram structures of the soil nutrients was spherical, the ratio of nugget/sill of soil available potassium, SOM, soil alkali hydrolysable nitrogen was $0.399,0.746$ and 0.696, respectively, which belonged to the middle intensity, the ratio of nugget/sill of soil available phosphorus was 0.759 , which belonged to the low intensity. Spatial variation of soil available potassium was mainly caused by structural factors(parent material, terrain, climate, water table etc.), the structure variability of it took $60.1 \%$ of the total variability, the spatial variation of soil available phosphorus, SOM and soil alkaline hydrolysis nitrogen were mainly caused by random factors(fertilization, environmental pollution, cultivation measures, management etc.), the random variability of them took $75.9 \%$, $74.6 \%, 69.6 \%$ of the total variability, respectively, the spatial correlation

Please use the following format when citing this chapter:

Yang, Y., Zhu, J., Tong, X. and Wang, D., 2009, in IFIP International Federation for Information Processing, Volume 293, Computer and Computing Technologies in Agriculture II, Volume 1, eds. D. Li, Z. Chunjiang, (Boston: Springer), pp. 125-134. 
distance of SOM, soil alkaline hydrolysis nitrogen, soil available potassium and soil available phosphorus was $44.898 \mathrm{~m}, 45.191 \mathrm{~m}, 57.691 \mathrm{~m}$ and 23.328 $\mathrm{m}$, respectively.

Keywords: soil nutrients, spatial variability, GIS, precision agriculture

\section{INTRODUCTION}

Spatial variability of soil characteristics benefits to comprehend the relationship between soil and environment, solve the problems about agriculture and resource management. With the rapid development of precision agriculture, the analysis of spatial distribution pattern of soil nutrients is becoming a focus and getting more and wide attention. To analyze spatial variability and pattern of soil nutrients is not only the foundation of practicing precision agriculture, but also is necessary for realizing the sustainable development of agriculture. In recent years, kriging techniques have been widely applied in soil, ecological, and environmental sciences to analyze the spatial patterns and variability of all kinds of properties. Some examples of kriging in soil nutrients include studies by some researcher(Gallardo, 2003; Ouyang et al., 2006; Guerra, et al., 2007; Li Rui, et al., 2007). However, the spatial distribution pattern of soil nutrients characteristics in the wheat field of the typical alluvial soil area has been rarely reported. Therefore, we chose a wheat field for revealing the spatial variability of soil available potassium, soil available phosphorus, SOM, soil alkali hydrolysable nitrogen, for estimating soil typical properties and mapping them, and for planning rational fertilization schemes.

\section{MATIERIALS AND METHOD}

\subsection{Experimental field and sampling laboratory treatment}

The study was conducted in a typical alluvial soil area in the YellowHuaihe Rivers Plain region, experiment site was carried out at the field $\left(117^{\circ}\right.$ $04.130^{\prime} \mathrm{E}, 36^{\circ} 42.979^{\prime} \mathrm{N}$ ), covering approximately $3 \mathrm{hm}^{2}$, located on the north shore of Xiaoqing River in Shandong province, P.R. China. This study area belonged to the warm temperate zone of half moist monsoon climate region, the terrain slope was high in the south and low in the north, and the soil was sandy clay loam. 104 soil samples were collected from the surface 
layer $(0-20 \mathrm{~cm})$ in June of 2007. Basically according to gridding sampling, each soil sampling was collected as a composite of 4 samples taken from the soil surface within a $5 \mathrm{~m}$ circular area centered on each site. All soil samples were analyzed for soil nutrients by the key laboratory of plant nutrient and fertilizer in Shandong province, soil $\mathrm{pH}$ was measured in 1:2.5(soil: water) suspensions with a $\mathrm{pH}$ meter, soil available potassium was measured using flame photometric method, soil available phosphorus was analyzed with colorimetry, SOM was analyzed by potassium dichromate volumetric method, soil alkali hydrolysable nitrogen was measured by diffusion method, the concrete analysis process referred to the book "Analytical Methods for Soil and Agricultural Chemistry" (Lu Rukui, 1999).

\subsection{Descriptive statistics of collected data}

The descriptive statistical parameters and normality tests were calculated with SPSS (version 15.0) and Origin8.0. Kriging estimation were mapped within Surfer 8.0(Golden Software), ArcGIS8.3 and its extension of geostatistical analyst, spatial patterns of soil nutrients was visualized by the different classes. Location of each sample point was measured with a simple differential global positioning system (DGPS) to obtain latitude and longitude values, detailed records were made on the coordinates of the sampling sites, these were converted to a metric coordinates. Sampling site geo-positions were transformed into ".shp" format, which is compatible with ArcView, and underwent ArcGIS8.3 projection transformation into plane coordinates, based on meters. The sampling map was used for geostatistical analysis.

The analysis of normality test is the prerequisite of kriging interpolation to calculate the real values of semivariogram. The study carried on the normality test of the soil nutrients content with P-P normal probability plot, there is the normality about the soil available phosphorus and the SOM, the soil available potassium and the soil alkaline hydrolysis nitrogen after logarithm transformation. So soil nutrients concentrations passed the P-P normal probability plot, which suggested that normality test had been achieved and could basically meet the requirements of geostatistical analysis(Yang Yujian et al., 2005; Li Xiaoyan, 2007). We calculated some parameters of 104 sampling data such as classical statistics (arithmetic means, standard deviation, coefficient of variation, minimum and maximum, median, skewness and kurtosis). Descriptive statistics of the measured properties are presented in Table1.

There are three classes about coefficient of variation(C.V.), weak variation $(\mathrm{C} . \mathrm{V} .<0.1)$, medium variation $(\mathrm{C} . \mathrm{V} .=0.1-1.0)$, strong variation (C.V.>1.0). Coefficient of variation of soil nutrients content, respectively (Yang Yujian, et al., 2005) . C.V. of soil available potassium content, SOM 
content and soil alkali hydrolysable nitrogen content, was $0.237,0.136$, 0.246 , respectively, which belonged to the medium variation. C.V. of the soil available phosphorus content was 15.285 , belonged to the strong variation. Table 1 showed that the study illustrated the characteristics of soil nutrients rather than the regional characteristics of the whole study area. This means that they cannot illustrate the spatial structural characteristics and the random variation of soil nutrients. Consequently, it is necessary to apply geostatistical methods together with GIS to solve this problem.

Table 1. Statistics characteristics of soil nutrients content

\begin{tabular}{cccccccccc}
\hline Item & Mean & Max & Min & Std. D & Var. & Skew. & Kur. & C.V. & Dis. \\
\hline SOM & 36.901 & 47.420 & 21.110 & 5.000 & 25.004 & -0.655 & 0.811 & 0.136 & N \\
N & 66.444 & 166.474 & 29.846 & 16.346 & 267.191 & 1.988 & 12.891 & 0.246 & $\operatorname{logN}$ \\
K & 287.475 & 483.433 & 119.435 & 68.286 & 4662.99 & 0.463 & 0.381 & 0.237 & $\operatorname{logN}$ \\
P & 4.23 & 293.13 & 18.77 & 28.790 & 828.860 & 5.676 & 45.981 & 15.285 & N \\
\hline
\end{tabular}

Notes: $\mathrm{N}$ stands for normal distribution; $\log \mathrm{N}$ stands for normal distribution after logarithm transformation

\subsection{Geostatistical analysis of study data}

A semivariogram, which graphs the semivariogram between spatially separate data points as a function of the distance is well documented to illustrate the spatial relationship of soil properties. It represents the relationship between the lag or any integral multiple of the sampling interval and the semivariogram. Semivariogram is theoretically shown as follow:

$$
\gamma(h)=\frac{1}{2 N(h)} \sum_{i=1}^{n}\left[Z\left(x_{i}+h\right)-Z\left(x_{i}\right)\right]
$$

Where: the $\gamma(h)$ quantity is function only of the increment $h, N(h)$ is the numbers of pairs separated by a distance $h, Z\left(x_{i}\right)$ and $Z\left(x_{i}+h\right)$ are the sampled values at location $x_{i}$ and $x_{i}+h$ for the analysis. The concepts and the theory of geostatistics referred to "Geostatistics in Environmental Science and its Application" (Zhang Renduo, 2005). Semivariograms revealed the spatial structure for all studied properties. The theoretical localized discontinuity or nugget, $\mathrm{C}_{0}$, is defined by the $\mathrm{y}$-intercept and is interpreted as variability due to experimental error, distributed effects below the sampling scale of the study, and other random effects. The corresponding value of the semivariogram at this point is termed the sill $\left(\mathrm{C}+\mathrm{C}_{0}\right)$ and represents the combination of a nugget effect and variability $(C)$ attributable to spatial dependence. The ratio of nugget value $\left(\mathrm{C}_{0}\right)$ to sill value $\left(\mathrm{C}_{0}+\mathrm{C}\right)$ is an important index for investigating the spatial structures of soil properties(Cation, 2001). In the study, there were only four soil properties for which we obtained reasonably accurate and effective predictions, including soil available potassium, soil available phosphorus, SOM and soil 
alkaline hydrolysis nitrogen. The semivariograms of soil nutrients exhibited excellent spatial structure, which could be well fitted with a spherical model, the parameters of spherical model were displayed in table 2 .

Table 2. Structure parameters of semivariogram and validation of soil nutrients model

\begin{tabular}{cccccccccccc}
\hline \multirow{2}{*}{ Var. Model } & \multirow{2}{*}{$\mathrm{C}_{0}$} & \multirow{2}{*}{$\mathrm{C}_{0}+\mathrm{C}$} & \multirow{2}{*}{ Range $(\mathrm{m})$} & $\mathrm{C}_{0} / \mathrm{C}_{0}+\mathrm{C}$ & \multicolumn{6}{c}{ Estimated error } \\
\cline { 7 - 11 } & & & & & & & $\mathrm{ME}$ & $\mathrm{RMSE}$ & $\mathrm{ASE}$ & $\mathrm{MSE}$ & $\mathrm{RMSSE}$ \\
$\mathrm{N}$ & $\mathrm{S}$ & 0.0270 & 0.0388 & 45.191 & 0.696 & 0.2592 & 15.15 & 14.11 & 0.001071 & 1.079 \\
$\mathrm{~K}$ & $\mathrm{~S}$ & 0.0183 & 0.0457 & 57.691 & 0.399 & -0.3519 & 52.61 & 52.35 & -0.01118 & 1.012 \\
$\mathrm{P}$ & $\mathrm{S}$ & 120.77 & 159.15 & 23.328 & 0.759 & 0.1904 & 14.69 & 14.84 & 0.01101 & 0.9925 \\
\hline
\end{tabular}

Note: S stands for spherical model.

The degree of spatial autocorrelation was described by the ratio of nugget and sill, if the ratio is smaller than $25 \%$, suggested the system has the intense spatial autocorrelation; if the ratio between $25 \% \sim 75 \%$, indicated the system has the medium spatial autocorrelation; if higher than $75 \%$ suggested the weak spatial autocorrelation. According to table 2, the ratio of nugget and sill of SOM, soil alkaline hydrolysis nitrogen, soil available potassium and soil available phosphorus were $0.746,0.696,0.399$ and 0.759 , respectively. Results showed there was the medium spatial autocorrelation of SOM, soil alkaline hydrolysis nitrogen, soil available potassium, the weak spatial autocorrelation of soil available phosphorus. Fig.1 shows the semivariogram model of soil available potassium, soil available phosphorus, SOM and soil alkaline hydrolysis nitrogen, respectively.

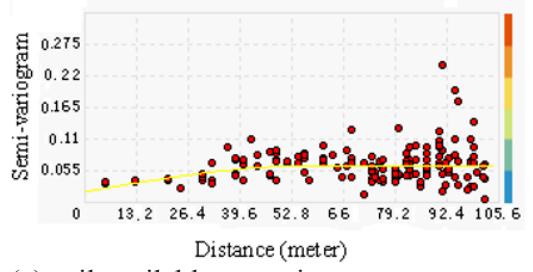

(a) soil available potassium

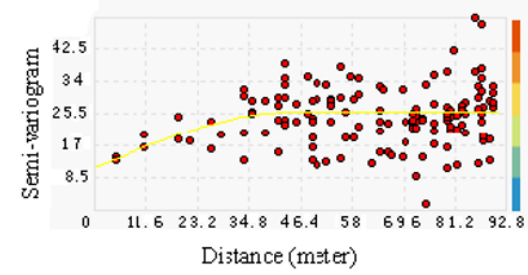

(c) soil SOM

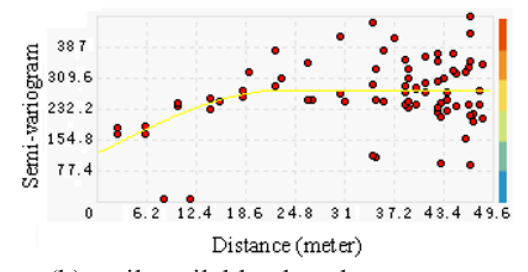

(b) soil available phosphorus

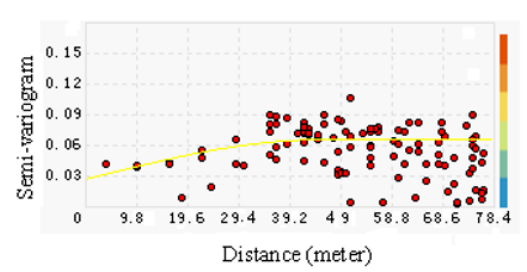

(d) soil alkaline hydrolysis nitrogen

Fig. 1. Semivariogram model of soil nutrients

A semivariaogram model is only an approximation of the spatial variability of the measured conditions, an indication of how well our semivariogram model approximates the experimental semivariogram is appraised by means of cross-validation procedure, cross-validation was used 
to obtain study by better resolving the spatial structure predicted and measured values, in this case a kriging estimation is made and compared with measurements. Thus, if for every one of the locations where we have a measured value, $Z\left(x_{i}\right)$, we estimate a value, $Z^{\prime}\left(x_{i}\right)$, then standard value of them were $Z_{1}\left(x_{i}\right)$ and $Z_{2}\left(x_{i}\right)$, then the expression of their average error, the average standard error, the root-mean-square error and the standardized rootmean-square error were

$$
\begin{aligned}
& M E=\frac{1}{N} \sum_{i=1}^{N}\left[Z\left(x_{i}\right)-Z^{\prime}\left(x_{i}\right)\right] \\
& M S E=\frac{1}{N} \sum_{i=1}^{N}\left[Z_{1}\left(x_{i}\right)-Z_{2}\left(x_{i}\right)\right] \\
& A S E=\sqrt{\frac{1}{N} \sum_{i=1}^{N}\left[Z^{\prime}\left(x_{i}\right)-\left(\sum_{i=1}^{N} Z^{\prime}\left(x_{i}\right)\right) / N\right]^{2}} \\
& R M S E=\sqrt{\frac{1}{N} \sum_{i=1}^{N}\left[Z\left(x_{i}\right)-Z^{\prime}\left(x_{i}\right)\right]^{2}} \\
& \text { RMSSE }=\sqrt{\frac{1}{N} \sum_{i=1}^{N}\left[Z_{1}\left(x_{i}\right)-Z_{2}\left(x_{i}\right)\right]^{2}}
\end{aligned}
$$

If $\mathrm{ME}$ (Mean standardized error) of the model approaches 0 , ASE(Average standard error) approaches RMSE(Root-mean-square error), MSE(Mean standard error) approaches

0 , RMSSE(Root-mean-square standardized error) approaches 1, which verifies the goodness of the fitted semivariogram models, estimated error of parameters of spatial pattern prediction referred in table 2. (Yang Yujian, et al., 2005; Zhang Wei, et al., 2007).

\section{RESULTS AND ANALYSIS}

Based on the fitted semiviariogram, we used ordinary kriging interpolation methods and acquired the spatial distribution maps of soil nutrients content at the field scale. Fig.2 shows the interpolation map revealed remarkable differences in the size, shape, and spatial distribution patterns about soil nutrients in the research area. Generally speaking, there were many factors affecting the spatial variation of soil nutrients, including structural factors (parent material, terrain, climate, water table etc.) and random factors (fertilization utilization, environmental pollution, cultivation measures, management etc.). From the above analysis, the study established 
the spatial distribution model of soil nutrients, the best fitted of spherical. Research results indicated that structural factors were the main factors of the spatial correlations of soil available potassium, the structure variability of it took $60.1 \%$ of the total variability, the spatial variation of soil available phosphorus, SOM and soil alkaline hydrolysis nitrogen were mainly caused by random factors, the random variability of them took $75.9 \%, 74.6 \%$, $69.6 \%$ of the total variability, respectively, the spatial correlation distance of SOM, soil alkaline hydrolysis nitrogen, soil available potassium and soil available phosphorus was $44.898 \mathrm{~m}, 45.191 \mathrm{~m}, 57.691 \mathrm{~m}$ and $23.328 \mathrm{~m}$. The paper also described the spatial distribution of soil nutrients characteristics. The spatial distribution pattern of soil nutrients(Fig.2), there is high value content of soil available potassium in the southeast and middle of the filed, soil available phosphorus in the middle of the filed, SOM in the southwest, northwest and middle of the field and soil alkaline hydrolysis nitrogen in the southeast and middle of the research site.
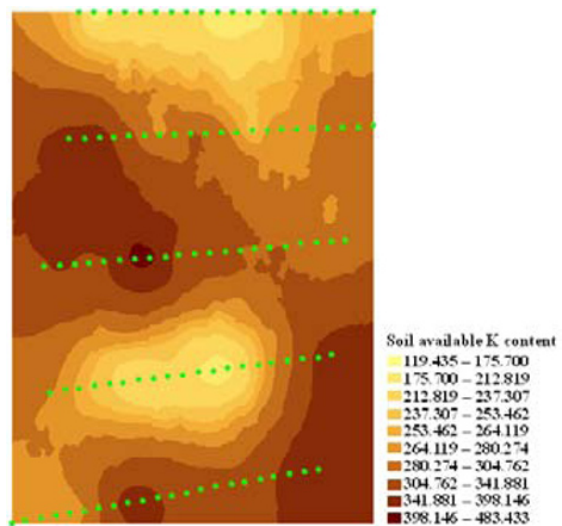

(a) soil available potassium

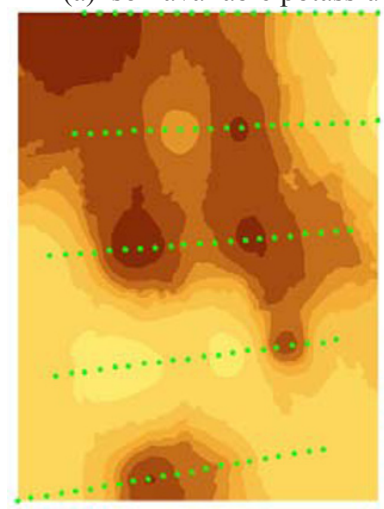

(c) soil SOM

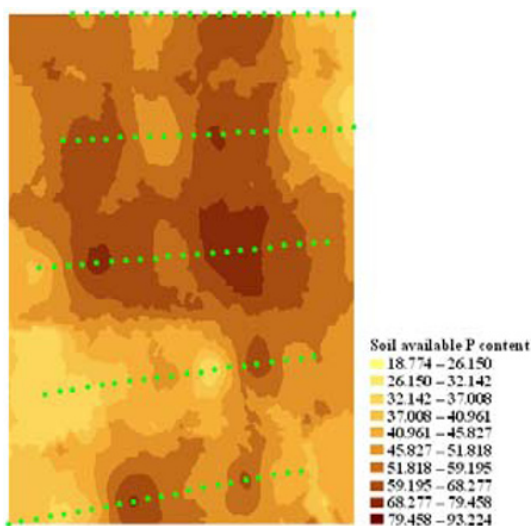

(b) soil available phosphorus

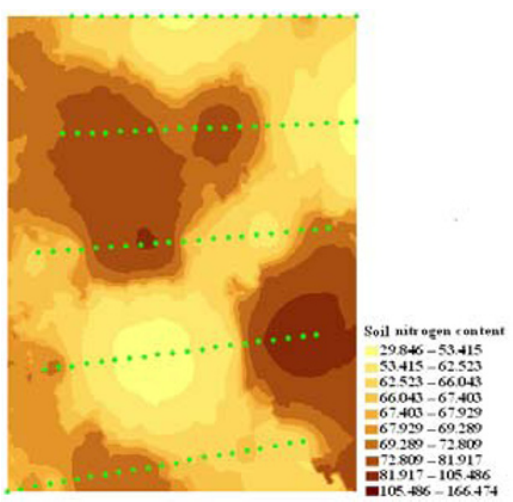

(d) soil alkaline hydrolysis nitrogen

Fig.2: Spatial distribution pattern of soil nutrients at the field scale 
There was the different soil characteristics in the different terrain, the spatial distribution of soil available potassium was relatively great affected by the micro-relief in the research site, as well as the absorption of crops, washing of soil nutrients and conditioner of soil $\mathrm{pH}$ in study field. There was a high value of SOM content in the southwest at the wheat field, where the irrigation canal flowed, in addition, organic matter stack became an important method of SOM increasing, according to the practical investigation, high organic fertilization also succeeded to the location of the history. Soil available phosphorus has two high value areas in the middle of the field, as the center, from inside to outside of its content presenting the distribution from high to low. From the scope of spatial scales, there was the low content in the southwest and northeast of the field, concerned with the closely irrigation canal, which affected the distribution of soil available phosphorus content, the distribution of it was concerned with present phosphate fertilization condition, for the small mobility of soil phosphorus, as well as the low fertilization utilization of the same season, resulting into the balance of soil phosphorus and the more phosphorus remains in soil, then forming the unevenly spatial distribution of soil phosphorus. The crop growth was affected nitrogen absorbing of crop, there is high value of soil alkaline hydrolysis nitrogen in the southeast and middle of the field, mainly for it. The investigation suggested the wheat growth is better than other places of the same field, the important factors of unbalance of soil alkaline hydrolysis nitrogen content was caused by the unbalance of fertilization, including nitrogen and organic fertilizer accumulation.

\section{CONCLUSIONS}

With the support of classical statistics, analysis results of 104 topsoil soil samples indicated that the average content of soil available potassium, available phosphorus, SOM and soil alkaline hydrolysis nitrogen was $287.476 \mathrm{~g} / \mathrm{kg}, 54.228 \mathrm{~g} / \mathrm{kg}, 36.901 \mathrm{~g} / \mathrm{kg}$ and $66.444 \mathrm{~g} / \mathrm{kg}$, respectively at the field scale. The paper also clarified the spatial distribution pattern of soil nutrients in the paper, research results indicated that structural factors were the main factors of the spatial autocorrelations of soil available potassium, the structure variability of it took $60.1 \%$ of the total variability, the spatial variation of soil available phosphorus, SOM and soil alkaline hydrolysis nitrogen were mainly caused by random factors, the random variability of them took $75.9 \%, 74.6 \%, 69.6 \%$ of the total variability, respectively. Results showed different spatial distribution pattern of different soil nutrients at the field scale, there is high value content of soil available potassium in the southeast and middle of the filed, soil available phosphorus in the middle of the filed, SOM in the southwest, northwest and middle of the field and 
soil alkaline hydrolysis nitrogen in the southeast and middle of the research site, the prediction results of model and actual fertilization are concordant.

\section{ACKNOWLEDGEMENTS}

This work was supported by the National Key Technology R\&D Program(2006BAD21B04-20) and Shandong Academy of Agricultural Science(SAAS) Youth Foundation Program(2005YQ021), we thank Professor. Chunjiang, Zhao (National Engineering Research Center for Information Technology in Agrticulture, NERCITA) and Professor. Guohui, Gan (Institute of Geographic Sciences and Natural Resources Research, CAS) for their constructive comments. We also wish to thank Dr. Shuyun, Liu; Dr. Xiaoyan, Zhang; Minghua, Shang; Fengyun, Wang; Wenjie, Feng; vice-professor quanting,Wan; Jia Zhao; Yanhong, Wang; Fujun, Wang and Xingde, Yin etc. for their data collection of S\&T Information Engineering Research Center of SAAS(P. R. China).

\section{REFERENCES}

Cation C, Cfitto A, Marcomini A,etal. Risk based characterization of contaminated industrial site using multivariate and geostatistical tools[J]. Environmental Pollution.2001,111:417427

Gallardo,A.Spatial variability of soil properties in a floodplain forest in northwest Spain.Ecosystems 2003, 6:564-576

Guerra, L C; Garcia y Garcia, A; Hook, J E; Harrison, K A; Thomas, D L; Stooksbury, D E; Hoogenboom, G. Irrigation water use estimates based on crop simulation models and kriging. Agricultural Water Management,2007, 89(3):199-207

Kong Xiangbin, Zhang Fengrong, Wang Ru. Spatial and temporal variation of soil nutrients in periurban region-a case study of Daxing District in Beijing city[J], Soils, 2004,36(6):636643(in Chinese)

Li Rui, Bai Youlu. Study on spatial variability of soil nutrient and crop yield[J]. Soil and Fertilizer Sciences in China,2007,3:40-42(in Chinese)

Li Xiaoyan, Wang Zongming, Zhang Bai et al. Geostatistical spatial structure analysis for crop productivity in Songnen Plain. System Sciences and Comprehensive Studies in Agriculture, 2007,23(1):45-51(in Chinese)

Lu RuKun. Analytical methods for soil and agricultural chemistry[M]. Beijing: China Agricultural Scientech Press. 1999. (in Chinese)

Ou yang,Y.,J.E.Zhang, and L.T.Ou.2006.Temporal and spatial distributions of sediment total organic carbon in an estuary river.J.Environ.Qual.35:93-100

Yang Yujian, Yang Jingsong. The trend variability of soil organic matter content in the salinity region of Yucheng city in Shandong Province[J]. Chinese Journal of Soil Science, 2005,36(5):647-651(in Chinese)

Zhang Renduo. Geostatistics in environmental science and its application. Beijing: Science Press, 2005 (in Chinese ) 
Zhang Wei, Chen Hongsong, Wang Kelin, et al. The heterogeneity of soil nutrients and their influencing factors in peak-cluster depression areas of karst region[J].Scientia Agricultura Sinica, 2006,39(9):1828-1835(in Chinese) 